\title{
CARACTERIZAÇÃO BIOMÉTRICA E FÍSICO-QUÍMICA DOS FRUTOS DE ACESSOS DE MANGA 'UBÁ'
}

\author{
JOSÉ CARLOS MORAES RUFINI ${ }^{2}$, EDUARDO RESENDE GALVÃO ${ }^{3}$, LUSINÉRIO PREZOTTI ${ }^{4}$, \\ MARCELO BARRETO DA SILVA ${ }^{5}$, RAFAEL AUGUSTO DA COSTA PARRELLA ${ }^{6}$
}

RESUMO - Este trabalho teve como objetivo avaliar a dissimilaridade genética de acessos de manga 'Ubá' na região leste de Minas Gerais, por meio da caracterização biométrica e físico-química dos frutos, visando a identificar materiais de interesse industrial para futuros trabalhos de melhoramento. Frutos de 67 acessos de mangueira Ubá provenientes do leste de Minas Gerais foram caracterizados, avaliando-se: massa da fruta, massa do endocarpo, relação polpa/endocarpo, diâmetro longitudinal, diâmetro transversal, sólidos solúveis totais, acidez total titulável, relação sólidos solúveis total/acidez total titulável e $\mathrm{pH}$. Os resultados foram avaliados por estatística descritiva, utilizando-se de medida de tendência central (média) e de variabilidade de dados (desvio-padrão). Foram realizadas análises estatísticas, utilizando-se das técnicas de agrupamento e medidas de dissimilaridade. Os frutos que apresentaram melhores características para o processamento foram os provenientes dos acessos $10 ; 22 ; 23 ; 24 ; 35 ; 38 ; 40 ; 42 ; 52 ; 55$ e 63 . A análise de agrupamento mostrou a formação de dois grupos de acessos com base nas características biométricas e físico-químicas dos frutos, o que demonstra variabilidade genética em mangueiras Ubá, no leste de Minas Gerais.

Termos para indexação: Mangifera indica L. melhoramento genético, diversidade.

\section{BIOMETRICAL AND PHYSICO-CHEMICAL CHARACTERIZATION OF FRUITS OF MANGO 'UBÁ’ ACCESSIONS}

\begin{abstract}
This study had as the main objective the evaluation of the genetic dissimilarity in mango 'Ubá' accessions in the eastern region of Minas Gerais by means of biometrical and physical-chemical characterization of the fruit in order to identify materials which have industrial interest for breeding studies. Fruits of 67 accessions were characterized by evaluation of fruit mass, endocarp mass, pulp/endocarp ratio, longitudinal diameter, transversal diameter, total soluble solids (TSS), titratable total acidity (TTA), TTS/TTA ratio and $\mathrm{pH}$. The results were evaluated by descriptive statistics using central trend (average) and variability of data (standard deviation). Statistical analyses were performed using the clustering techniques and dissimilarity measures. The fruits which have showed the best characteristics for the processing were the hits from the 10, 22, 23, 24, 35, 38, 40, 42, 52, 55 and 63. Cluster analysis demonstrated the formation of two groups of accesses in reference with the biometrical and the physical-chemical characterization of the fruit, which shows the genetic variability the eastern Minas Gerais' mango.
\end{abstract}

Index terms: Mangifera indica L., plant breeding, diversity.

\footnotetext{
${ }^{1}$ (Trabalho 100-10). Recebido em: 19-04-2010. Aceito para publicação em: 10-11-2010.

${ }^{2}$ Prof. Doutor em Fruticultura, Universidade Federal de São João Del Rei/UFSJ, CEP 35701-970. Sete Lagoas-MG. E-mail: rufini@ufsj.edu.br

${ }^{3}$ Prof. Doutor em Genética e Melhoramento de plantas, Universidade Vale do Rio Doce/UNIVALE, CEP 37200-000. Governador Valadares-MG. E-mail: eduadogalvao@univale.br

${ }^{4}$ Prof. Doutor em Entomologia, Instituto Federal de Educação, Ciência e Tecnologia do Espírito Santo. CEP 29660-000. Santa TeresaES. E-mail: lusineriop@ifes.edu.br

${ }^{5}$ Prof. Doutor em Fitopatologia, UFES, Centro Universitário Norte do Espírito Santo. CEP 29931-220. São Mateus-ES. E-mail: barretofito@uol.com.br

${ }^{6}$ Pesquisador Doutor em Genética e Melhoramento de plantas, EMBRAPA CNPMS, CEP 35701-970. Sete Lagoas-MG. E-mail: parrella@cnpms.embrapa.br
} 


\section{INTRODUÇ̃̃O}

O Brasil produz anualmente 1.154 mil toneladas de manga, cultivadas em praticamente todos os estados brasileiros, com destaque para a Bahia, São Paulo, Pernambuco e Minas Gerais, responsáveis por $84 \%$ da produção nacional (IBGE, 2008). Entretanto, mesmo com recentes aumentos nas exportações brasileiras, o mercado externo não é o principal destino da produção. A maior parte destina-se ao mercado interno de frutas frescas, com pequena utilização pela indústria.

Em Minas Gerais, há grande diversidade de mangueiras, especialmente a variedade Ubá, muito cultivada na zona da mata e leste do estado. Segundo Gonçalves et al. (1998), esta variedade é indicada tanto para o consumo in natura quanto para a industrialização.

Preferencialmente, o mercado industrial de processamento de frutas prioriza a matéria-prima que possua características como alto rendimento em polpa, alto teor de sólidos solúveis e ausência de fibras. Segundo Chitarra e Chitarra (2005), em manga, a proporção entre a polpa, a casca e o endocarpo é fortemente influenciada pelo fator varietal, e a relação SST/ATT é uma das formas mais utilizadas para a avaliação do sabor, sendo mais representativa que a medição isolada de açúcares ou da acidez.

Benevides et al. (2008), em trabalho realizado na Zona da Mata Mineira para determinar a qualidade da manga Ubá destinada ao processamento industrial, concluíram que a polpa apresentou valores para $\mathrm{pH}$, sólidos solúveis, acidez, relação sólidos solúveis/ acidez e açúcares totais dentro dos padrões exigidos pela legislação.

Alguns trabalhos têm sido conduzidos no intuito de obter materiais promissores para o melhoramento genético da mangueira. É notória a seleção de genótipos mediante avaliação da diversidade genética e caracterização de cultivares (NUNES et al., 1991; FONSECA et al., 1994; GALLI et al., 2008), aplicação da biologia molecular (RAJWANA et al., 2008;SANTOS et al., 2008) e análise da qualidade organoléptica dos frutos (RIBEIRO et al., 2007; VERGARA-VALENCIA et al., 2007; WANG et al., 2007; RIBEIRO et al., 2008; FARAONI et al., 2009; MONTALVO et al., 2009).

O presente estudo teve cromo objetivo realizar a caracterização biométrica e físico-química de acessos de manga 'Ubá' na região leste de Minas Gerais, visando a identificar materiais de interesse industrial para futuros trabalhos de melhoramento.

\section{MATERIAL E MÉTODOS}

O trabalho foi realizado na região leste de Minas Gerais, onde foram pré-selecionadas mangueiras adultas e produtivas da variedade Ubá, oriundas de propagação sexuada com mais de 20 anos de idade. Com base no histórico e observação das plantas, realizou-se uma pré-seleção de 67 acessos distribuídos em oito municípios: Galileia (19), Governador Valadares (13), Era Nova (13), Engenheiro Caldas (12), Alpercata (3), Itambacuri (3), Conselheiro Pena (2) e Aimorés (2). As coordenadas geográficas de cada acesso foram definidas pelo sistema GPS (Global Position System) - DATUM Cartográfico SAD-69.

O experimento foi conduzido nas safras de 2005 e 2006. As plantas demarcadas foram acompanhadas a partir do florescimento, sendo as frutas amostradas quando se apresentavam fisiologicamente maduras, evidenciada pela intensificação da coloração amarela da casca. Foram coletadas, aleatoriamente em cada acesso, as frutas situadas na posição mediana da planta e distribuídas ao longo do perímetro da copa. Utilizou-se um coletor mecânico acoplado em uma haste de $4 \mathrm{~m}$ de altura para a obtenção da fruta sem sua queda ao solo. As frutas foram acondicionadas em sacos plásticos, identificadas e conduzidas para o Laboratório de Pós-Colheita da Universidade Vale do Rio Doce (UNIVALE), em Governador Valadares, para que se processassem as análises biométricas e físico-químicas. Os dados relativos à massa da fruta $(\mathrm{MF})$ e à massa do endocarpo (ME) foram determinados em uma balança de precisão, sendo os resultados expressos em (g). A relação polpa/endocarpo foi obtida dividindo-se o resultado da diferença entre a massa da fruta e conforme determinado por Chitarra e Chitarra (2005). O diâmetro longitudinal (DL) e o diâmetro transversal (DT) foram determinados com paquímetro digital, e os resultados, expressos em (mm). O teor de sólidos solúveis totais (SST), medido em porcentagem, foi aferido utilizando-se de um refratômetro digital. A acidez total titulável (ATT) foi determinada por titulometria, sendo os resultados expressos em $\%$ de ácido cítrico. A relação SST/ATT e o $\mathrm{pH}$ da polpa foram avaliados com o uso de peagâmetro.

O delineamento experimental utilizado para as análises biométricas e físico-químicas das frutas correspondeu ao inteiramente casualizado, considerando-se 67 acessos (fator de variação) e quatro repetições por acesso. Foram colhidas 20 frutas de cada acesso e deste total foram retiradas quatro amostras de cinco frutas cada. Os dados foram avaliados por estatística descritiva, utilizando-se de medida de tendência central (média) e de variabilidade de dados 
(desvio-padrão). Inicialmente, utilizaram-se as seguintes medidas de posição: mínimo, mediana, média, máximo e, como medida de dispersão, utilizou-se o desvio-padrão para descrever os caracteres avaliados. Após a padronização dos dados, foi realizada a análise de agrupamentos através do método hierárquico de Ward, utilizando como medida de dissimilaridade a distância euclidiana média. As análises estatísticas foram realizadas, utilizando-se do programa estatístico GENES - Aplicativo Computacional em Genética e Estatística (CRUZ, 2001).

\section{RESULTADOS E DISCUSSÃO}

A massa da fruta de 67 acessos de manga 'Ubá' variou de 91,5 a 182,25 g, com média de 128,7 $\mathrm{g}$ (Tabela 1). O acesso 64 foi o que mais se destacou em relação à massa da fruta. Analisando frutas da mesma variedade, Galli et al. (2008) observaram, para a massa da fruta, 138,75 g, e Faraoni et al. (2009), 143,8 g, em mangas provenientes da região da Zona da Mata em Minas Gerais. As variações percebidas para a massa da fruta podem ser decorrentes de diversos fatores internos e externos. Além dos fatores intrínsecos à planta, como o genético, outros podem ser decisivos sobre a qualidade da fruta, destacandose o número de frutos por planta e a competição entre órgãos em desenvolvimento (FONFRÍA et al. 1996). Entretanto, dos muitos fatores externos que podem influenciar no crescimento do fruto, a disponibilidade de água e a temperatura exercem maior efeito no desenvolvimento e qualidade (ALBRIGO, 1975), como foi observado na variabilidade da massa da fruta da mangueira Ubá decorrente de diferentes microrregiões de cultivo no leste de Minas Gerais.

Para a massa do endocarpo, observou-se variação de $8,25 \mathrm{~g}$ a 21,0 g, e média de 14,2 $\mathrm{g}$ (Tabela 1). Entre os 67 acessos de manga 'Ubá', o acesso 23 apresentou a menor massa do endocarpo, com média de 8,25 g. Em trabalho visando a caracterizar a biometria de sementes da variedade 'Ubá', Borges et al. (1999) obtiveram média de 12,49 g para massa do endocarpo da manga produzida na Zona da Mata mineira e Galli et al. (2008) verificaram média de 28 $\mathrm{g}$ para a massa do endocarpo desta mesma variedade, cultivada em Pindorama-SP. Para que uma variedade de manga tenha boa aceitação comercial, a massa do endocarpo não deve exceder a $10 \%$ da massa da fruta (YOUNG; SAULS, 1979; DONADIO, 1980). Dos 67 acessos estudados no leste de Minas Gerais, $31,3 \%$ apresentaram para a massa do endocarpo índices inferiores a $10 \%$ da massa da fruta, permitindo identificar os acessos mais promissores ao aproveitamento industrial e a futuros trabalhos de melhoramento genético.

Analisando a relação polpa/endocarpo, foi observada média de 8,25 para a relação polpa/ endocarpo em massa, com valores mínimos de 4,93 para o acesso 65 e máximo de 15,67 para o acesso 23 (Tabela 1). Segundo Chitarra e Chitarra (2005), a relação polpa/endocarpo é importante em frutas como a manga, onde o endocarpo aumenta durante o crescimento do fruto, influenciando seu peso específico, todavia essa relação pode provir da influência varietal. Notadamente, os acessos que apresentam maiores índices da relação polpa/ endocarpo, predispõem-se especialmente para fins industriais por apresentarem maior rendimento em polpa. No presente estudo, verificaram-se índices superiores à média para a relação polpa/endocarpo em $47 \%$ dos acessos de manga 'Ubá'.

Com relação ao diâmetro do fruto, observouse, em média, $71,58 \mathrm{~mm}$ e $56,66 \mathrm{~mm}$ para diâmetro longitudinal e transversal, respectivamente (Tabela 1). Foi encontrado valor mínimo de $59,24 \mathrm{~mm}$ e máximo $81,75 \mathrm{~mm}$ para diâmetro longitudinal e mínimo de $50,88 \mathrm{~mm}$ e máximo de $65,03 \mathrm{~mm}$ para diâmetro transversal. Faraoni et al. (2009), avaliando o diâmetro da manga Ubá em cultivo orgânico na Zona da Mata mineira, observaram valores de $75 \mathrm{~mm}$ e $57 \mathrm{~mm}$ para o diâmetro longitudinal e transversal, respectivamente. Esses valores assemelham-se ao tamanho do fruto produzido no leste de Minas Gerais.

Os resultados de sólidos solúveis totais (SST) encontrados para a polpa da manga 'Ubá' variaram de $9,3 \%$ a 19,5\%, com média de $14,24 \%$ (Tabela 1 ), inferior ao teor encontrado para a mesma variedade na região da Zona da Mata mineira por Gonçalves et al. (1998), Benevides et al. (2008) e Faraoni et al. (2009), de $16,8 \%, 18 \%$ e $19,3 \%$, respectivamente. É provável que esses frutos tenham sido avaliados em estádio mais avançado de maturação. Os frutos dos genótipos de manga Ubá provenientes do leste de Minas Geras tiveram os teores de sólidos solúveis determinados no mesmo dia da colheita, no ponto de maturação fisiológica. Já Neves et al. (2008) observaram valores de $8,9 \%$ para a cultivar Haden e 9,1\% para a cultivar Tommy Atkins em frutas colhidas em estádio fisiologicamente maduras. De acordo com Chitarra e Chitarra (2005), em geral, o teor de sólidos solúveis varia com espécies, variedades, estádios de maturação e clima, com valores médios situados entre $8 \%$ e $14 \%$. Em estudo com a cv. Ataulfo, no México, Montalvo et al. (2009) verificaram incremento de $17,47 \%$ no teor de SST durante o período de armazenamento da manga e Wang et al. (2007), 
com a cv Tainong, em Hainan/China, observaram o mesmo comportamento para o SST, com ascensão e pico no quinto dia de armazenamento.

Os valores de acidez total titulável (ATT), encontrados em polpa dos 67 acessos de manga Ubá, variaram de $0,26 \%$ a $1,8 \%$ de acido cítrico com média de $0,54 \%$ (Tabela 1 ). Valores semelhantes foram observados por Gonçalves et al. (1998), de 0,54\% de ácido cítrico e Benevides et al. (2008), de 0,56\% para a mesma variedade. Entretanto, Faraoni et al. (2009) verificaram valor inferior para a acidez em polpa de manga Ubá sob cultivo orgânico de $0,40 \%$ de acido cítrico. À medida que o fruto amadurece, concomitantemente o valor da acidez é reduzido e o teor de sólidos solúveis totais aumentado, influenciando positivamente no incremento da relação SST/ATT.

A relação SST/ATT variou de 5,08 a 57,7, com média de 32,88 (Tabela 1). Trabalhando com a mesma variedade, Gonçalves et al. (1998) e Benevides et al. (2008) obtiveram, para a relação SST/ATT, médias de 31,47 e 34,52, respectivamente, próximas da verificada neste trabalho, enquanto a relatada por Faraoni et al.(2009) foi superior $(48,2)$, cujo valor foi atribuído pelos autores ao estádio mais avançado de amadurecimento do fruto.

A determinação do $\mathrm{pH}$ e da acidez fornece dados valiosos na apreciação do estado de conservação de um produto alimentício. Os valores de $\mathrm{pH}$ observados por Gonçalves et al. (1998), Benevides et al. (2008) e Faraoni et al. (2009), para a variedade Ubá, compreenderam a faixa entre 4,1 e 4,3, valores que corroboram os resultados obtidos neste trabalho quando foi observado para $\mathrm{pH}$, média de 4,18 (Tabela 1). Para os 67 acessos de manga Ubá, verificaram-se valores mínimos de 2,6 e máximo de 4,8 . Os resultados estão em conformidade com a legislação vigente, pois a polpa deve apresentar pH abaixo de 4,5, para garantir sua conservação sem a necessidade de tratamento térmico muito elevado, para que assim não coloque em risco a sua qualidade. Entretanto, 20 acessos $(1 ; 4 ; 7 ; 13 ; 26 ; 31 ; 36 ; 41 ; 44$; $45 ; 46 ; 51 ; 53 ; 54 ; 56 ; 57 ; 58 ; 59 ; 60$ e 62$)$ registraram valores com $\mathrm{pH}$ acima ou abaixo dos parâmetros que regulamentam os padrões para polpa de manga no Brasil, os quais não devem ser indicados para o processamento industrial.

A contribuição relativa dos caracteres para a dissimilaridade genética de acessos de manga 'Ubá', no leste de Minas Gerais, está apresentada na Tabela 2. Os caracteres adotados neste trabalho podem ser considerados representativos, uma vez que foram eficientes na análise de dissimilaridade e posterior agrupamento dos acessos. O maior índice observado foi de $27,5 \%$ para a massa da fruta, seguido da relação SST/ATT (13,5\%), diâmetro transversal da fruta $(12,3 \%)$ e relação polpa/endocarpo (10,4\%). A acidez e o $\mathrm{pH}$ foram os caracteres com menor variabilidade na análise da dissimilaridade dos acessos de manga Ubá, com valores de 5,4\% e 4,5\%, respectivamente.

A análise de agrupamento permitiu a elaboração do dendrograma hierárquico, apresentado na Figura 1, a partir de caracteres biométricos e físicoquímicos dos frutos de 67 acessos de manga Ubá. Nota-se a formação de dois grupos distintos, sendo um constituído por 12 acessos com distância genética inferior a $25 \%$ entre subgrupos e menor que $1 \%$ dentro destes subgrupos, onde se pode verificar materiais bastante similares geneticamente nos agrupamentos formados pelos acessos $(17 ; 15 ; 56 ; 47 ; 49$ e 6$),(43 \mathrm{e}$ $39)$ e $(21 ; 9 ; 33$ e 11). Outro grupo constituído por 55 acessos apresentou similaridade acima de $99 \%$ para os agrupamentos $(24 ; 55 ; 63 ; 1 ; 38$ e 23$),(54 ; 13 ; 62$ e 51$),(53 ; 4 ; 64$ e 34$),(32 ; 30 ; 58$ e 7$),(31 ; 50 ; 48$; 61 e 3$),(19$ e 5$),(26 ; 10 ; 52$ e 35$),(25 ; 41$ e 37$),(8 ;$ 46 e 44$),(27 ; 16 ; 45$ e 29$),(28 ; 14 ; 57$ e 36$),(22 ; 42$ e 40), (18; 67 e 65$),(12 ; 60$ e 59) e (2; 66 e 20$)$. 
TABELA 1 - Características biométricas e físico-químicas de 67 acessos de manga 'Ubá’ no leste de Minas Gerais.

\begin{tabular}{|c|c|c|c|c|c|c|c|c|c|}
\hline $\begin{array}{c}\text { Acessos/ } \\
\text { local }\end{array}$ & $\begin{array}{l}\text { Massa do } \\
\text { fruto }(\mathrm{g})\end{array}$ & $\begin{array}{l}\text { Massa do } \\
\text { endocarpo }(\mathrm{g})\end{array}$ & $\begin{array}{l}\text { Relação polpa/ } \\
\text { endocarpo }\end{array}$ & $\begin{array}{l}\text { Diâmetro } \\
\text { longitudinal } \\
\text { (mm) }\end{array}$ & $\begin{array}{l}\text { Diâmetro } \\
\text { transversal } \\
(\mathrm{mm})\end{array}$ & $\begin{array}{c}\text { SST } \\
\%\end{array}$ & $\begin{array}{c}\text { ATT } \\
\% \text { acido } \\
\text { cítrico }\end{array}$ & SST/ATT & $\mathrm{pH}$ \\
\hline $1 / \mathrm{G}$ & 101.9625 & 12.75 & 7.00 & 65.9 & 52.2475 & 15.225 & 0.38875 & 39.16399 & 4.65 \\
\hline $2 / \mathrm{G}$ & 106.705 & 13 & 7.21 & 69.2225 & 52.4575 & 14.625 & 0.5815 & 25.15047 & 3.9925 \\
\hline $3 / \mathrm{G}$ & 101.9925 & 11.75 & 7.68 & 66.8525 & 52.1125 & 14.65 & 0.61675 & 23.75355 & 3.525 \\
\hline $4 / \mathrm{G}$ & 131.8575 & 11.75 & 10.22 & 77.425 & 56.525 & 15.125 & 0.6515 & 23.21566 & 3.2375 \\
\hline $5 / \mathrm{G}$ & 109.4525 & 13 & 7.42 & 72.6925 & 54.0525 & 13.925 & 0.78825 & 17.66572 & 4.1775 \\
\hline $6 / \mathrm{G}$ & 97.0425 & 12.875 & 6.54 & 65.7875 & 51.295 & 15.325 & 0.6995 & 21.90851 & 4.115 \\
\hline $7 / \mathrm{G}$ & 105.8925 & 12.225 & 7.66 & 67.775 & 53.3925 & 15.15 & 0.56425 & 26.8498 & 4.605 \\
\hline $8 / \mathrm{G}$ & 136.875 & 13.75 & 8.95 & 77.2975 & 57.7275 & 11.85 & 1.047 & 11.31805 & 4.0775 \\
\hline 9/G & 103.6025 & 12.5 & 7.29 & 66.8275 & 52.585 & 16.3 & 0.36 & 45.27778 & 4.38 \\
\hline $10 / \mathrm{G}$ & 159.5975 & 15.25 & 9.47 & 76.465 & 61.2475 & 13.9 & 0.3545 & 39.21016 & 4.3875 \\
\hline $11 / \mathrm{G}$ & 140.06 & 20.5 & 5.83 & 74.9525 & 57.175 & 12.675 & 0.4055 & 31.25771 & 4.535 \\
\hline $12 / \mathrm{G}$ & 126.66 & 10.75 & 10.78 & 64.265 & 57.85 & 11.8 & 0.568 & 20.77465 & 4.105 \\
\hline $13 / \mathrm{G}$ & 150.2925 & 11.5 & 12.07 & 68.0775 & 52.755 & 11.625 & 0.5325 & 21.83099 & 4.5575 \\
\hline $14 / \mathrm{G}$ & 102.13 & 12.75 & 7.01 & 76.24 & 58.98 & 13.25 & 0.69875 & 18.96243 & 4.0075 \\
\hline $15 / \mathrm{G}$ & 136.6825 & 11.5 & 10.89 & 69.3675 & 57.445 & 13.65 & 0.58025 & 23.52434 & 4.545 \\
\hline $16 / \mathrm{G}$ & 107.0875 & 10.25 & 9.45 & 67.52 & 51.73 & 14.45 & 0.3095 & 46.68821 & 4.5425 \\
\hline $17 / \mathrm{G}$ & 153.9 & 14.75 & 9.43 & 81.725 & 61.2475 & 12.35 & 0.556 & 22.21223 & 4.0725 \\
\hline $18 / \mathrm{G}$ & 146.36 & 13.5 & 9.84 & 78.865 & 58.045 & 15.3 & 0.735 & 20.81633 & 4.195 \\
\hline $19 / \mathrm{G}$ & 96.5925 & 12.25 & 6.89 & 65.3225 & 50.8725 & 16.8 & 0.3515 & 47.79516 & 3.6325 \\
\hline 20/EC & 146.0425 & 14.75 & 8.90 & 75.6175 & 58.4075 & 12.725 & 0.44775 & 28.41988 & 4.4 \\
\hline 21/EC & 152.1175 & 14 & 9.87 & 77.38 & 59.7225 & 14.025 & 0.50675 & 27.67637 & 4.305 \\
\hline 22/EC & 125.2725 & 16.5 & 6.59 & 73.7075 & 56.0325 & 14.2 & 0.4115 & 79 & 3.57 \\
\hline $23 / \mathrm{EC}$ & 137.525 & 8.25 & 15.67 & 77.7275 & 58.29 & 13.9 & 0.42375 & 32.80236 & 4.4325 \\
\hline $24 / \mathrm{CP}$ & 129.45 & 13 & 8.96 & 73.4975 & 57.71 & 14.075 & 0.41275 & 34.10055 & 4.3425 \\
\hline $25 / \mathrm{CP}$ & 138.25 & 12.75 & 9.84 & 77.565 & 58.15 & 13 & 0.96925 & 13.41243 & 3.635 \\
\hline $26 / \mathrm{I}$ & 142.75 & 10.75 & 12.28 & 75.375 & 59.375 & 10.75 & 1.4095 & 7.626 & 2.6 \\
\hline 27/I & 121.75 & 12 & 9.15 & 68.925 & 57.665 & 12.95 & 0.31475 & 41.14376 & 4.4125 \\
\hline 28/I & 123.5 & 13 & 8.50 & 69.7525 & 55.23 & 13.7 & 0.36275 & 37.76706 & 4.33 \\
\hline 29/EC & 136.57 & 10.75 & 11.70 & 75.3525 & 63.84 & 12.7 & 0.56675 & 22.40847 & 4.3525 \\
\hline $30 / \mathrm{EC}$ & 109.63 & 12.25 & 7.95 & 69.9775 & 53.245 & 13.125 & 0.51025 & 25.72268 & 4.3025 \\
\hline $31 / \mathrm{EC}$ & 158.1075 & 12.75 & 11.40 & 79.965 & 60.745 & 12.425 & 1.26125 & 9.851338 & 2.925 \\
\hline $32 / \mathrm{EC}$ & 114.325 & 13.75 & 7.31 & 68.6225 & 54.92 & 12.925 & 0.88875 & 14.5429 & 4.035 \\
\hline $33 / \mathrm{EC}$ & 158.795 & 13 & 11.22 & 81.5925 & 61.25 & 12.575 & 0.6725 & 18.69888 & 4.09 \\
\hline $34 / \mathrm{EC}$ & 102.5125 & 12 & 7.54 & 66.2325 & 54.95 & 16.8 & 0.38325 & 43.83562 & 4.22 \\
\hline $35 / \mathrm{EC}$ & 126.615 & 19.5 & 5.49 & 73.935 & 56.2975 & 15.475 & 0.3455 & 44.79016 & 4.165 \\
\hline $36 / \mathrm{AL}$ & 157.0025 & 12.25 & 11.82 & 79.995 & 59.77 & 13.825 & 0.3045 & 45.4023 & 4.59 \\
\hline 37/AL & 109.935 & 11.75 & 8.36 & 68.455 & 53.505 & 14.625 & 0.524 & 27.91031 & 4.3075 \\
\hline 38/AL & 102.4825 & 12.25 & 7.37 & 65.9475 & 52.5425 & 12.65 & 0.407 & 31.08108 & 4.29 \\
\hline 39/EC & 124 & 20 & 5.20 & 73.3 & 56.1425 & 13.675 & 0.70175 & 19.487 & 4.01 \\
\hline $40 / \mathrm{GV}$ & 119 & 16.25 & 6.32 & 59.24 & 55.95 & 16.575 & 0.2875 & 57.65217 & 3.76 \\
\hline $41 / \mathrm{GV}$ & 120.4 & 12.25 & 8.83 & 66.575 & 55.11 & 19.5 & 0.3735 & 52.20884 & 4.56 \\
\hline $42 / \mathrm{GV}$ & 121.05 & 13 & 8.31 & 68.62 & 54.2225 & 17.05 & 0.32475 & 52.50192 & 4.385 \\
\hline $43 / \mathrm{GV}$ & 124.75 & 13.5 & 8.24 & 68.3825 & 55.355 & 15 & 0.44425 & 33.76477 & 4.4125 \\
\hline $44 / \mathrm{GV}$ & 121.25 & 13.75 & 7.82 & 68.415 & 54.995 & 13.65 & 0.41 & 33.29268 & 4.55 \\
\hline $45 / \mathrm{GV}$ & 174.25 & 12.75 & 12.67 & 78.83 & 61.92 & 17.25 & 0.33425 & 51.60808 & 4.67 \\
\hline $46 / \mathrm{GV}$ & 128 & 13 & 8.85 & 71.9875 & 56.005 & 14.55 & 0.3055 & 47.62684 & 4.55 \\
\hline $47 / \mathrm{GV}$ & 123 & 13 & 8.46 & 68.8575 & 54.8025 & 16.3 & 0.29025 & 56.15848 & 4.205 \\
\hline $48 / \mathrm{GV}$ & 140.75 & 13.1 & 9.74 & 76.065 & 56.985 & 13.15 & 0.68825 & 19.10643 & 4.0625 \\
\hline $49 / \mathrm{GV}$ & 148.75 & 19.5 & 6.63 & 74.09 & 58.2125 & 13.875 & 0.4505 & 30.79911 & 4.395 \\
\hline $50 / \mathrm{GV}$ & 135.25 & 18.5 & 6.31 & 70.4625 & 57.3 & 15.225 & 0.388 & 39.23969 & 3.695 \\
\hline $51 / \mathrm{GV}$ & 138.125 & 12.5 & 10.05 & 71.7675 & 56.905 & 12.9 & 1.83 & 7.04918 & 2.92 \\
\hline $52 / \mathrm{GV}$ & 155 & 14.7 & 9.54 & 73.8175 & 61.3175 & 17.075 & 0.37025 & 46.11749 & 4.135 \\
\hline 53/AI & 118.75 & 17.25 & 5.88 & 68.105 & 56.4775 & 13.85 & 0.32375 & 42.77992 & 4.78 \\
\hline
\end{tabular}




\begin{tabular}{|c|c|c|c|c|c|c|c|c|c|}
\hline 54/AI & 117.5 & 12.825 & 8.16 & 75.6425 & 55.6575 & 9.3 & 1.82875 & 5.085441 & 2.9175 \\
\hline $55 / \mathrm{EN}$ & 103.75 & 15 & 5.92 & 66.9425 & 51.975 & 15.925 & 0.29325 & 54.3052 & 4.61 \\
\hline $56 / \mathrm{EN}$ & 115 & 17.25 & 5.67 & 67.075 & 53.675 & 16.225 & 0.35475 & 45.73643 & 4.7 \\
\hline $57 / \mathrm{EN}$ & 143 & 16.5 & 7.67 & 69.65 & 56.25 & 17.75 & 0.43625 & 40.68768 & 4.6 \\
\hline $58 / \mathrm{EN}$ & 141.75 & 12.5 & 10.34 & 71.4225 & 59.9675 & 13.8 & 0.31575 & 43.70546 & 4.7525 \\
\hline $59 / \mathrm{EN}$ & 115 & 15.25 & 6.54 & 70.015 & 54.6475 & 13.7 & 0.305 & 44.91803 & 4.5725 \\
\hline $60 / \mathrm{EN}$ & 149.25 & 20 & 6.46 & 71.405 & 60.6725 & 15.725 & 0.3225 & 48.75969 & 4.77 \\
\hline $61 / \mathrm{EN}$ & 147.25 & 18.5 & 6.96 & 73 & 59.79 & 13.7 & 0.405 & 33.82716 & 4.5475 \\
\hline $62 / \mathrm{EN}$ & 143.5 & 18.5 & 6.76 & 72.1425 & 62.8425 & 13.875 & 0.26 & 53.36538 & 4.8125 \\
\hline $63 / \mathrm{EN}$ & 137.5 & 17.5 & 6.86 & 71.5325 & 58.82 & 13.9 & 0.3775 & 36.82119 & 4.2875 \\
\hline $64 / \mathrm{EN}$ & 182.25 & 20.75 & 7.78 & 78.285 & 65.0375 & 15.65 & 0.6025 & 25.9751 & 4.01 \\
\hline $65 / \mathrm{EN}$ & 124.5 & 21 & 4.93 & 70.0275 & 56.4725 & 13.45 & 0.32 & 42.03125 & 4.2875 \\
\hline $66 / \mathrm{EN}$ & 111.5 & 16.25 & 5.86 & 68.125 & 54.3075 & 12.825 & 0.4375 & 29.31429 & 3.9525 \\
\hline 67/EN & 91.5 & 14.75 & 5.20 & 59.765 & 53.425 & 14.25 & 0.35525 & 40.1126 & 3.575 \\
\hline $\begin{array}{l}\text { Desvio- } \\
\text { padrão }\end{array}$ & 20.05 & 2.88 & 2.12 & 4.95 & 3.24 & 1.75 & 0.3 & 13.38 & 0.5 \\
\hline Mínimo & 91.5 & 8.25 & 4.93 & 59.24 & 50.87 & 9.3 & 0.26 & 5.08 & 2.6 \\
\hline Máximo & 182.25 & 21 & 15.67 & 81.725 & 65.03 & 19.5 & 1.8 & 57.7 & 4.8 \\
\hline Mediana & 126.62 & 13 & 7.95 & 71.41 & 56.47 & 13.9 & 0.41 & 33.29 & 4.30 \\
\hline Média & 128.7 & 14.2 & 8.35 & 71.58 & 56.66 & 14.24 & 0.54 & 32.88 & 4.18 \\
\hline
\end{tabular}

Galileia (G), Engenheiro Caldas (EC), Conselheiro Pena (CP), Itambacuri (I), Alpercata (AL), Governador Valadares (GV), Aimorés (AI) e Era Nova (EN).

TABELA 2 - Contribuição relativa dos caracteres para a dissimilaridade genética de acessos de manga 'Ubá' no leste de Minas Gerais.

\begin{tabular}{lcc}
\hline \multicolumn{1}{c}{ Variável } & S.j & Valor em \% \\
\hline Massa da fruta & 9792108.0 & 27.5 \\
Massa do endocarpo & 3513056.0 & 9.8 \\
Relação polpa/endocarpo & 3705314.0 & 10.4 \\
Diâmetro transversal & 4376046.0 & 12.3 \\
Diâmetro longitudinal & 2842520.0 & 8.0 \\
SST & 3095162.0 & 8.7 \\
ATT & 1924538.0 & 5.4 \\
SST/ATT & 4831274.0 & 13.5 \\
pH & 1593894.0 & 4.5 \\
\hline
\end{tabular}

S.j - valor da contribuição relativa dos caracteres 


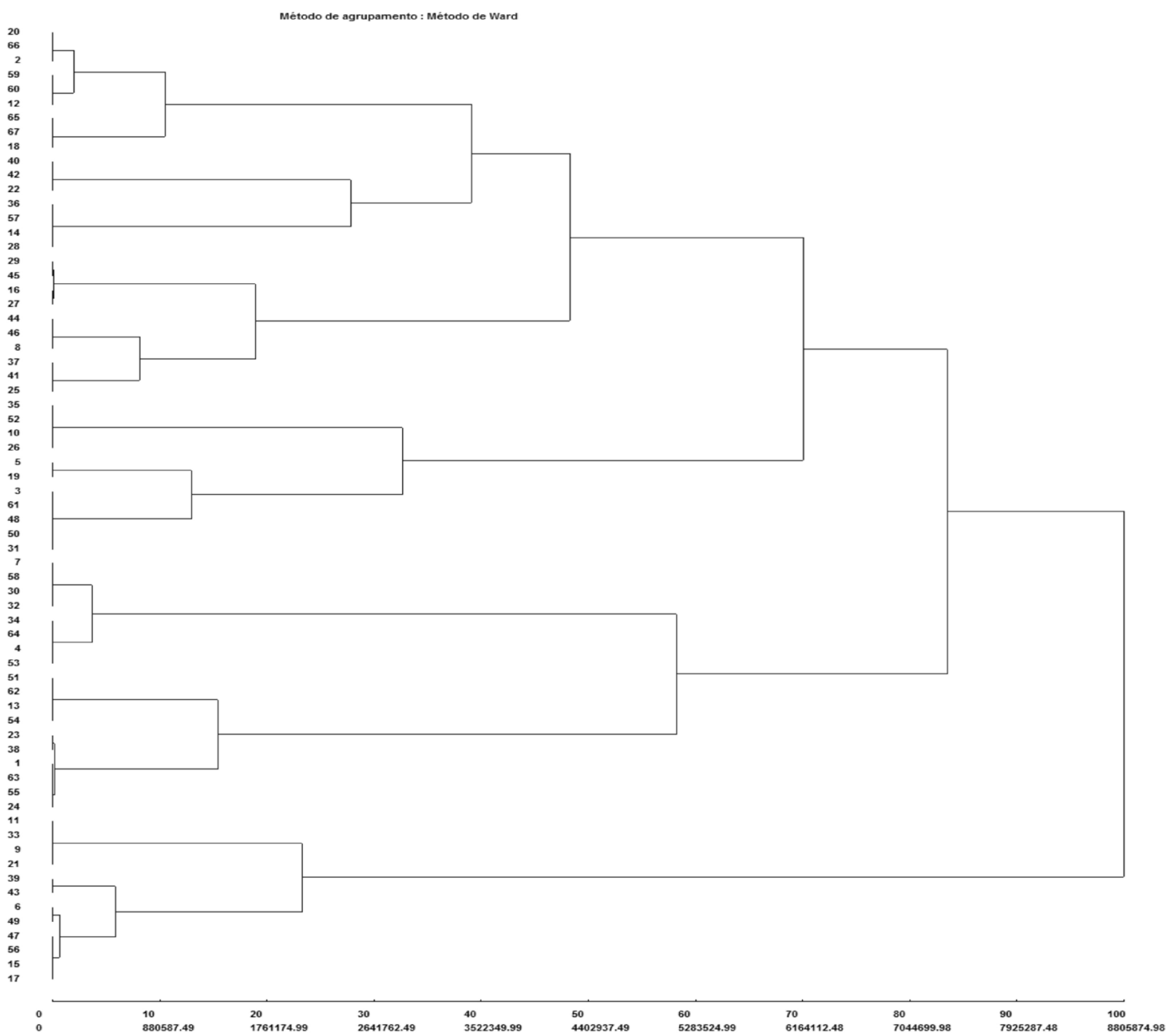

FIGURA 1 - Dendrograma de similaridade de 67 acessos de manga 'Ubá' obtidos a partir de caracteres biométricos e físico-químicos dos frutos através do método de agrupamento de Ward, baseado na distância euclidiana média. 


\section{CONCLUSÕES}

1-Os frutos de mangueira Ubá que apresentam melhores características para o processamento são os provenientes dos acessos $10 ; 22 ; 23 ; 24 ; 35$; $38 ; 40 ; 42 ; 52 ; 55$ e 63.

2-Massa da fruta, relação sólidos solúveis/ acidez, diâmetro transversal da fruta e relação polpa/ endocarpo são as características que apresentaram maior variabilidade dos acessos de mangueira Ubá.

3-A diversidade genética existente na mangueira Ubá, no leste de Minas Gerais, propicia disponibilizar materiais para futuros trabalhos de melhoramento genético e implantação de banco de germoplasma.

\section{AGRADECIMENTOS}

Ao apoio financeiro da Fundação de Amparo à Pesquisa do Estado de Minas Gerais (FAPEMIG).

\section{REFERÊNCIAS}

ALBRIGO, L. G. Water relations and citrus fruit quality. In: SAULS, J. W.; JACKSON, L. K. Water realations. Gainiville: University of Florida Fruit/ Crops Department, 1975. p. 41-48.

IBGE. Produção Agrícola Municipal 2008: Malha municipal digital do Brasil: situação em 2008. Disponível em: <http://www.ibge. gov.br/estadosat/temas.php?sigla=mg \&tema=lavourapermanente2008> Acesso em: 30 jan. 2009.

BENEVIDES, S. D.; RAMOS, A.M.; STRINGHETA, P.C.; CASTRO V.C. Qualidade da manga e polpa da manga Ubá. Ciência Tecnologia de Alimententos, Campinas, v. 28, n. 3, set. 2008.

BORGES, C.A.M.; SIQUEIRA, D.L.de; DIAS, D.C.F.dos; CARDOSO, A.A. Caracterização e correlação biométricas de sementes das mangueiras 'Espada' e 'Ubá'. Revista Ceres, Viçosa, MG, v.46. n.264. p. 219-229, 1999.

CHITARRA, A. B.; CHITARRA, M. I. F. Pós-colheita de frutos e hortaliças: fisiologia e manuseio. Lavras: UFLA, 2005. 783 p.
CRUZ, C.D. Programa genes: versão Windows, aplicativo computacional em genética e estatística. Viçosa: UFV, 2001. 648p.

DIAS, I.A. dos S. Análises multidimensionais. In: ALFENAS, A.C. (Ed.). Eletroforese de isoenzimas e proteínas afins: fundamentos e aplicações em plantas e microrganismos. Viçosa-MG: UFV, 1998. p.405-475.

DONADIO, L.C. Cultura da mangueira. Piracicaba: Livroceres, 1980. 72p.

FARAONI, A.S.; RAMOS, A.M.; STRINGHETA, P.C. Caracterização da manga orgânica cultivar ubá. Revista Brasileira de Produtos Agroindustriais, Campina Grande, v.11, n.1, p.9-14, 2009.

FONFRÍA, M. A.; ORENGA,V. A.; ALCAINA, M. A.; FERRER, M. J.; ROMERO, V. E. Citros: desenvolvimento e tamanho final do fruto. Porto Alegre: Cinco Continentes, 1996. 102 p.

FONSECA, N.; SILVA, S. de O. e; SAMPAIO, J.M.M. Caracterização e avaliação de cultivares de manga na região do Recôncavo Baiano. Revista Brasileira de Fruticultura, Jaboticabal, v.16, n.3, p.29-45, 1994.

GALLI, J.A.; MICHELOTTO, M.D.; SIVEIRA, L.C.P.; MARTINS, A.L.M. Qualidade de mangas cultivadas no Estado de São Paulo. Bragantia, Campinas, v.67, n.3, p.791-797, 2008.

GONÇALVES, N. B.; CARVALHO, V.D.; GONÇALVES, J.R.A.; COELHO, S.R.M.; SILVA, T.G. Caracterização física e química dos frutos de cultivares de mangueira (Mangifera indica L.). Ciência e Agrotecnologia, Lavras,v. 22, n. 1, p. 72-78, jan./ mar. 1998.

MONTALVO, E.; ADAME, Y.; GARCI, H.S.; TOVAR, B.; MATA, M. Changes of sugars, b-carotene and firmness of refrigerated Ataulfo mangoes treated with exogenous ethylene. Journal of Agricultural Science, Cambridge, v. 147, p. 193-199, 2009.

NEVES, L.C.; BENEDETTE, L.M.; SILVA, V.X da; PRILL, M. A. de S.; ROBERTO, S.R.; VIEITES, R.L. Qualidade pós-colheita de mangas, não refrigeradas, e submetidas ao controle de ação do etileno. Revista Brasileira de Fruticultura, Jaboticabal, v.30, n.1, p.129-137, 2008 
NUNES, R.F.M.; SAMPAIO, J.M.M.; RODRIGUES, J.A. Comportamento de cultivares de mangueira (Mangifera indica L.) sob irrigação na Região do vale do São Francisco. Revista Brasileira de Fruticultura, Jaboticabal, v.15, n.3, p.129-137, 1991

RAJWANA, I.A.; TABBASAM, N.; MALIK, A.U.; MALIK, S.A.; UR-RAHMAN, M.; ZAFAR, Y. Assessment of genetic diversity among mango (Mangifera indica L.) genotypes

using RAPD markers. Scientia Horticulturae, Amsterdam, v. 117, p. 297-301, 2008

RIBEIRO, S.M.R.; BARBOSA, L.C.A.; QUEIROZ, J.H.; KNODLER, M.; SCHIEBER, A. Phenolic compounds and antioxidant capacity of Brazilian mango (Mangifera indica L.) varieties. Food Chemistry, London, v. 110, n. 3, p. $620-626,2008$.

RIBEIRO, S.M.R.; QUEIROZ, J.H.; QUEIROZ, M.E.L.R. de; CAMPOS, F.M.; SANTANA, H.M.P. Antioxidant in mango (mangifera indica L.) pulp. Plant Foods for Human Nutrition, v.62, n.1, p.13-17, 2007.
SANTOS, C.A.F.; LIMA NETO, F.P.; RODRIGUES.M.A.; COSTA, J.G.da.Similaridade genética de acessos de mangueira de diferentes origens geográficas avaliadas por marcadores AFLP. Revista Brasileira de Fruticultura, Jaboticabal, v.30, n.3, p.736-741, 2008

VERGARA-VALENCIA, N.; GRANADOSPÉREZ, E.; AGAMA-ACEVEDO, E.; TOVAR, J.; RUALES, J.; BELLO-PÉREZ, L.A. Fibre concentrate from mango fruit: Characterization, associated antioxidant capacity and application as a bakery product ingredient. Food Science and Technology, London, v. 40, p. 722-729, 2007.

YOUNG, T.W.; SAULS, J.W. The mango industry in Florida. Gainesville: University of Florida, 1979. 70 p. (Bulletin, 189)

WANG, J.; WANG, B.; JIANG, W.; ZHAO, Y. Quality and Shelf Life of Mango (Mangifera Indica L. cv. 'Tainong') Coated by Using Chitosan and Polyphenols. Food Science and Technology International, Beijing, v. 13, p. 317-322, 2007. 